\title{
La educación se mueve en el territorio. Estrategias locales de aprendizaje-servicio
}

\section{Sara Maroto Sales}

Servicios de Educación, Diputación de Barcelona

\section{Resumen}

Este artículo es un relato de la trayectoria de la Diputación de Barcelona en relación al despliegue realizado, durante la última década, de una política de apoyo y difusión del aprendizaje-servicio combinando diferentes iniciativas y formulaciones que cristalizan en la adopción y puesta en funcionamiento de una línea de apoyo a los entes locales para la formulación y puesta en funcionamiento de estrategias locales de aprendizaje-servicio. Se pone en contexto la realidad y se explica la evolución de las medidas y de las acciones que se han emprendido: desde un momento inicial, de difusión de la metodología de aprendizaje-servicio en alianza con agentes clave del ámbito educativo hasta un momento actual, de integración del aprendizaje-servicio en un contexto más amplio, de política pública local. Por el camino, el aprovechamiento de las ventanas de oportunidad que plantea la actual ordenación curricular en nuestro país y el desarrollo de alianzas con agentes diversos, dentro y fuera de nuestra organización. Se concluye con la convicción de que el municipio tiene un rol especialmente importante en el desarrollo de proyectos con metodología de aprendizaje-servicio, aportando calidad a partir del conocimiento de la realidad del territorio. También se hace evidente que el aprendizaje-servicio impulsa la movilización del entorno en su labor de apoyo al éxito educativo.

\section{Palabras clave}

Política educativa local, éxito educativo, entorno educativo, apoyo y acompañamiento, articulación. 


\title{
Education moves in territory. Service-learning local strategies
}

\begin{abstract}
This article aims to provide an account of the efforts made by the Barcelona Provincial Council, over the past decade, to implement a policy supporting and disseminating service-learning. The Council combined different initiatives and formulations that crystallised in the adoption and implementation of a line of support to local entities for the formulation and application of local service-learning strategies. In this article, we place this policy in context and we discuss how the various measures and actions that have been undertaken have evolved, starting from the initial phase, when service-learning methodology was first promoted in alliance with key educational agents, up to the present time, when service-learning is being integrated into a broader context, that of local public policy. We also discuss how we took advantage of certain windows of opportunity offered by the current curricular structure in Spain and how partnerships were developed with various agents, both inside and outside our organisation. The paper concludes with the conviction that the municipality has a particularly important role to play in the development of service-learning projects, contributing to ensuring quality based on the knowledge of the territory's reality. The paper also evidences that service-learning promotes the mobilisation of the environment in its work to support educational success.
\end{abstract}

\section{Keywords}

Local educational policy, educational success, educational environment, support and accompaniment, articulation. 


\section{Contexto: mundo local y política educativa}

El aprendizaje-servicio es aprendizaje significativo. La aportación de significado, en procesos de aprendizaje, tiene que ver con la capacidad de relacionar el concepto que se aprende con el contexto físico y social donde el individuo la aplicará: el entorno. A la hora de plantear estos procesos en clave de servicio público hay que situar el contexto institucional de la política educativa.

El desarrollo de políticas educativas en España se ha caracterizado por una descentralización interrumpida del sistema educativo. El gobierno central retiene competencias mínimas en la ordenación de las enseñanzas y ha trasladado hacia las comunidades autónomas (regiones) el grueso esencial de competencias. En Cataluña, el gobierno de la Generalitat se constituye en la Administración educativa: competencias en el desarrollo de las enseñanzas y desarrollo y gestión del sistema de escolarización. Sin embargo, esta descentralización no ha alcanzado el tercer nivel de gobierno, formado por los gobiernos locales. A diferencia de otras referencias de ámbito Europeo, los municipios han sido bastante abandonados tanto por el legislador estatal como por el autonómico. En este sentido, podemos hablar de un sistema altamente centralizado donde la presencia más o menos significativa del territorio ha dependido más de las sensibilidades, capacidades y destrezas de los municipios que de unas leyes que hayan abierto puertas (Tarabini,
Bonal y Plandiura, 2017).

A pesar de no disponer de un mandato legal claro ni de un sistema de financiación en correspondencia, el mundo local ha asumido, como gobierno de proximidad, la participación en la gestión de la función educativa en los respectivos territorios destinando importantes esfuerzos y volúmenes de gasto público. Las políticas educativas municipales han alcanzado dimensión en la planificación del entorno en clave educativa, en la adopción de programas y medidas de acompañamiento a la escolaridad, de orientación y transición educativa.

El mundo local aporta al sistema de políticas educativas su dimensión más territorial mientras que las sucesivas administraciones educativas en Cataluña han hecho hincapié en la escuela y en el aula, a menudo con poca sensibilidad por la dimensión comunitaria o el refuerzo de la corresponsabilidad en la necesaria gobernanza multinivel de los asuntos educativos en las sociedades actualesí.

La articulación de programas y servicios públicos en el ecosistema de gobiernos locales en Cataluña se hace en dos niveles: municipal y supramunicipal. En el nivel supramunicipal encontramos el gobierno de las diputaciones en territorios (provincias o demarcaciones) que agrupan una colectividad de municipios. La demarcación de Barcelona está compuesta por 311 municipios, incluida la ciudad capital, y representa el $24 \%$ del total de la superficie de Cataluña y el $74,4 \%$ del total de la población catalana (más de

Maroto, S. (2018). La educación se mueve en el territorio. Estrategias locales de aprendizaje-servicio. RIDAS, Revista Iberoamericana de Aprendizaje Servicio, 6, 19-32. DOI10.1344/RIDAS2018.6.4 
cinco millones y medio de personas).

El gobierno de la Diputación de Barcelona está formado por alcaldes, alcaldesas, concejales y concejalas municipales. Las diputaciones no disponen de competencias materiales (estas son de los municipios) sino de competencias funcionales: asistencia y cooperación con los municipios para el desarrollo de sus políticas y para la prestación de servicios públicos de calidad.

Esta asistencia y cooperación se concreta en la transferencia de recursos técnicos (trabajos de asesoramiento y consultoría para el diseño de intervenciones públicas), económicos (ayudas, fondos de prestación y créditos) y materiales (prestación directa de bienes, equipos, servicios y actividades). También puede coordinar servicios municipales y organizar servicios públicos de carácter supramunicipal.

Las diputaciones son, pues, una administración "de" y "para" los municipios. La cultura de trabajo entre ambos niveles se fundamenta en el respeto a la autonomía local y en el trabajo en red entre los gobiernos locales y la Diputación para construir un programa de actuación conjunto, de acuerdo con los principios de eficiencia y de economía de la acción pública. La finalidad es compartir objetivos, crear más bienestar en todo el territorio y mejorar la calidad de los servicios, para lo cual la competencia de los profesionales y la difusión de las buenas prácticas son imprescindibles.

Desde la Gerencia de Servicios de
Educación de la Diputación se articula el espacio de asistencia y cooperación en el desarrollo de las políticas educativas locales en Barcelonaii. El Plan de Actuación de Mandato 20152019 se formula a partir de concebir el éxito educativo como éxito personal a lo largo de toda la vida, con una perspectiva de intervención pública integral, reforzando el papel de los ayuntamientos e implicando a los agentes educativos del territorio. La finalidad es favorecer la cohesión social y la calidad de vida de los pueblos y ciudades mediante la mejora de la educación. Estos objetivos se articulan a través de tres líneas principales de trabajo que reúnen todos los proyectos de apoyo técnico, económico y material a los entes locales de la provincia:

- Desarrollo del Servicio Catalán de Educación: reforzar la acción responsable de los gobiernos locales en el desarrollo del Servicio de Educación de Cataluñaiii para garantizar una educación de calidad.

- Mejora del éxito con el entorno: aumentar el éxito educativo con servicios educativos básicos orientados a las personas desde la adaptación de la especificidad local.

- Reducción del abandono escolar prematuro: consolidar políticas y programas para garantizar itinerarios personales y transiciones educativas a lo largo de la vida.

\section{Evolución del aprendizaje- servicio en la Diputación de Barcelona}

La experiencia de la Diputación de

Maroto, S. (2018). La educación se mueve en el territorio. Estrategias locales de aprendizaje-servicio. RIDAS, Revista Iberoamericana de Aprendizaje Servicio, 6, 19-32. DOI10.1344/RIDAS2018.6.4 
Barcelona en relación al aprendizajeservicio dibuja una trayectoria que comienza con la publicación de la Guía metodológica del Aprendizaje-Servicio (2008) y camina hacia la articulación de esta propuesta metodológica en la política educativa local, con el territorio como espacio de arraigo y de trabajo propio de esta administración. De forma sintética, podemos dividir este camino en tres etapas, coincidentes con los tres últimos períodos de mandato local:

1) Participación en el diseño e impulso de la metodología ApS (2008-2012)

A partir de la experiencia con actuaciones educativas desde el entorno, como los proyectos intergeneracionales, los espacios de refuerzo socioeducativo y la oferta educativa local, se considera que el ApS es una metodología idónea para poner al servicio de estos y otros proyectos que, desde el territorio, buscan el éxito educativo. La Diputación se implica en el Centro Promotor de AprendizajeServicio. La finalidad principal de este Centro es promover el estudio, la difusión y el desarrollo de proyectos de aprendizaje-servicio. Desde su creación, formaron parte del grupo coordinador la Fundación Catalana del Esplai, el Instituto de Ciencias de la Educación y el Grupo de Investigación en Educación Moral (GREM) de la Universidad de Barcelona, el Área de Educación de la Diputación de Barcelona y la Fundación Jaume Bofill. Esta metodología educativa contiene los ingredientes suficientes de educación y de territorio, este último como espacio de actuación propio de la Diputación. Permite conectar espacios de educación formal y reglada, con espacios de educación no formal e informal. Permite conectar, también, las necesidades de aprendizaje en el seno de los centros educativos con las necesidades de transformación social propias de cada territorio. Esta estrategia queda recogida en la Guía metodológica del Aprendizaje Servicio (2008), un documento de trabajo elaborado con el objetivo de poner en valor el potencial del aprendizaje servicio como metodología educativa a promover e impulsar desde el territorio, así como facilitar elementos para su puesta en marcha desde la política educativa local. Este material se convierte, también, un elemento importante de comunicación y difusión del compromiso que la Gerencia de Servicios de Educación toma en relación al ApS. Asimismo, sienta las bases para sistematizar un proceso de apoyo específico para este ámbito, en el que se reclama la participación corresponsable de tres agentes fundamentales de la comunidad educativa: ayuntamientos, centros educativos y entidades. Esta acción sumada a otras de carácter formativo y de sensibilización, pone al alcance de los entes locales la capacitación del territorio para recoger, fortalecer y desarrollar actuaciones con metodología ApS.

2) Integración del apoyo ApS en el programa de acompañamiento a la escolaridad (2012-2015)

Viendo las potencialidades del aprendizaje-servicio como metodología educativa en el ámbito municipal, se encaja en el Programa de Acompañamiento a la Escolaridadiv, que

Maroto, S. (2018). La educación se mueve en el territorio. Estrategias locales de aprendizaje-servicio. RIDAS, Revista Iberoamericana de Aprendizaje Servicio, 6, 19-32. DOI10.1344/RIDAS2018.6.4 
ofrece un marco de trabajo más amplio vinculado al éxito escolar y educativo de toda la población. Este programa está formado por tres ejes:

1)Colaboración con centros educativos: ámbito de trabajo que pone énfasis en la creación y/o acompañamiento de actuaciones que desde el territorio facilitan el aprendizaje curricular propio de los centros educativos. 2)Apoyo a la función educativa de las familias: pone la mirada en el necesario apoyo a las familias para desarrollar sus funciones parentales-marentales. 3)Entorno: apoyo a actuaciones fuera del horario lectivo. Trabaja para la movilización de todos los recursos del entorno, para ponerlos al servicio de los procesos de aprendizaje en todos los tiempos y espacios vitales. Es en este eje donde se ubica el ApS.

En este marco de trabajo se inicia la construcción de un proyecto que identifica el ApS como un instrumento para la mejora del éxito educativo.

3) Pilotaje y generación de Estrategias Locales de ApS (2015 a 2019).

En paralelo a esta tarea de Diputación, en 2015 el Departamento de Enseñanza de la Generalidad de Cataluña se inclina por la creación de un proyecto curricular y, por tanto, de obligado cumplimiento para todo el alumnado de 30 o 40 de Educación Secundaria Obligatoria: el servicio comunitario. El proyecto tiene como finalidad última garantizar que los estudiantes, a lo largo de su trayectoria escolar, experimenten y protagonicen acciones de compromiso cívico, aprendan en el ejercicio activo de la ciudadanía, y pongan en juego sus conocimientos y capacidades al servicio de la comunidad, con unos objetivos con marcado componente territorial. Ante la propuesta, la Diputación de Barcelona hace un último giro respecto al aprendizaje-servicio resituándolo como una estrategia de articulación de esta metodología educativa en las políticas educativas locales. La estrategia se concreta en dos líneas básicas, una de trabajo con los ayuntamientos y otra de trabajo a nivel interno en la Gerencia de Servicios de Educación. La primera, para acompañar y facilitar herramientas a los entes locales en su colaboración en el desarrollo de los proyectos de servicio comunitario y vincular éste a otras prácticas con metodología ApS que se estén desarrollando en el municipio. En esta misma línea se hace una importante iniciativa para el trabajo interáreas, con el fin de establecer una estrategia global de apoyo y trabajo de la metodología ApS en el territorio.

Diferentes áreas y servicios de la Diputación de Barcelona se muestran interesadas en el aprendizaje servicio: Bienestar Social, Bibliotecas, Espacios Naturales, Medio Ambiente, Oficina del Plan Joven y Deportes. Se visualiza el ApS como una oportunidad para poner en práctica el trabajo transversal, movilizar agentes y recursos del territorio, trabajar el aprendizaje en valores y reforzar el trabajo en red de toda la comunidad educativa, no sólo de centros educativos y entidades sino también de las administraciones. La segunda línea propone la incorporación de la metodología ApS en programas y/o proyectos propios de la Gerencia de Servicios de Educación, con el objetivo de identificar acciones para las que la 
incorporación de la metodología ApS represente un valor añadido, como por ejemplo en la educación especial o el aprendizaje por competencias.

\section{Configuración de un nuevo espacio de soporte municipal}

La estrategia relativa al ApS se lleva a cabo a partir de tres líneas básicas de provisión: formación, comunicación, apoyo técnico y otras acciones.

\section{Formación}

Una buena manera de poner al alcance de todos los entes locales la información y conocimiento sobre el ApS y su valor en la política educativa local, ha sido a través de los espacios de formación y seminarios de trabajo impulsados desde la Gerencia de Servicios de Educación.

Los cursos de formación realizados hasta el momento han tenido como finalidad conocer y analizar en profundidad las oportunidades que esta metodología educativa aporta en el espacio de gobierno municipal. Se han explicado los fundamentos y conceptos básicos y los principios pedagógicos, se han mostrado experiencias exitosas, se han buscado fórmulas de arraigo en el territorio atendiendo al encaje de esta propuesta con otras propuestas educativas y espacios de participación, planificación o programación existentes (Consejos Escolares Municipales, Planes Educativos de Entorno, Proyectos Educativos de Ciudad, entre otros). Estos cursos se han ofrecido a todos los municipios de la demarcación de Barcelona a través del Plan de
Formación de la Gerencia de Servicios de Educación, y se han llevado a cabo en los años 2012, 2015 y 2016.

Debe mencionarse de forma especial una jornada del año 2012 en la que se hizo una propuesta de encaje entre el aprendizaje-servicio y los Proyectos Educativos de Ciudad. La jornada se tituló "De la participación en la ciudad educadora. El aprendizaje servicio en el marco de los PEC" y se concibió como un espacio de sensibilización y formación sobre una posible y muy interesante estrategia de arraigo del ApS en el territorio y, aún más importante, de visualizarla como un instrumento facilitador del concepto de Ciudad Educadora.

También se han realizado seminarios de intercambio con otra finalidad, la de facilitar un espacio para el diálogo, reflexión y valoración de experiencias exitosas o con un alto grado de desarrollo. Estos seminarios sólo se han abierto a aquellos municipios con una clara estrategia de trabajo con la metodología ApS.

Los cursos de formación y seminarios han contado con la participación de 40 municipios, que representan el $41 ' 8 \%$ de la población de la demarcación de Barcelona (sin contar con Barcelona Ciudad $)^{\mathrm{v}}$.

\section{Comunicación}

La comunicación se entiende como una herramienta para dar a conocer a los ayuntamientos el apoyo que la Diputación de Barcelona ofrece en general y, concretamente, en relación al aprendizaje-servicio. 
Uno de los canales habituales es el boletín digital mensual, educ@diba, en el que se informa de todos los temas de interés para los ayuntamientos, entre ellos el ApS. Otra forma importante de comunicación es a través de la elaboración de material bibliográfico, como el caso de la guía citada anteriormente.

\section{Soporte técnico}

El objetivo final del soporte técnico es acompañar a los referentes de educación, técnicos y políticos, en la construcción de una estrategia de articulación del proyecto Servicio Comunitario y otras actuaciones con metodología ApS en la política educativa local. El apoyo se dirige a todos los entes locales para que puedan acompañar a los centros de educación secundaria y las entidades del municipio en el desarrollo del proyecto así como de otras actuaciones con metodología ApS en centros educativos de infantil, primaria, adultos o entidades del ocio, entre otros.

Desde que el Departamento de Educación de la Generalidad de Cataluña estableció la obligatoriedad de que todo el alumnado de $3^{\circ}$ o $4^{\circ}$ de ESO de Cataluña realice el proyecto Servicio Comunitario, este toma especial relevancia para los entes locales. Miles de alumnos deberán realizar un servicio a la comunidad. Los ayuntamientos se convierten en la pieza clave para encajar centro educativo y territorio y aportar todos los elementos para que este proyecto educativo se desarrolle con la máxima calidad.
El colaborador principal de la Diputación de Barcelona en el acompañamiento a los entes locales es el Centro Promotor de ApS. Hasta diciembre de 2017 esta entidad formaba parte de la Fundación Jaume Bofill. A partir de enero de 2018 funciona de forma independiente como una asociación sin ánimo de lucro.

Para que los ayuntamientos puedan recibir el apoyo técnico descrito, deben formalizar una solicitud a través del Catálogo de Servicios de la Diputación de Barcelona. El Servicio de Apoyo Municipal valida la concesión del apoyo. Si se admite, se contacta con el ayuntamiento para hacer una reunión de concreción de la demanda y elaboración de un plan de trabajo acordado por ambas partes. Este trabajo de modulación del soporte técnico permite a la Gerencia de Servicios de Educación establecer tres planos de intervención e integrar el ApS en un contexto más amplio, de política educativa municipal, ocupando una posición intermedia entre la planificación del tiempo y el espacio educativo y las intervenciones directas dentro y fuera escuela.

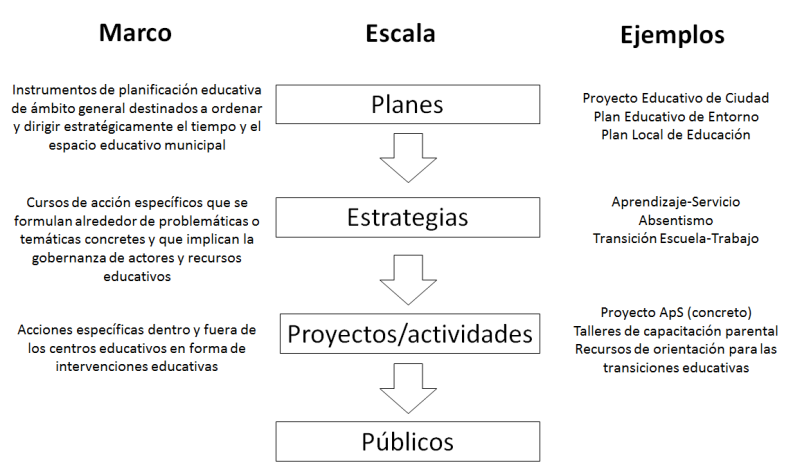

Maroto, S. (2018). La educación se mueve en el territorio. Estrategias locales de aprendizaje-servicio. RIDAS, Revista Iberoamericana de Aprendizaje Servicio, 6, 19-32. DOI10.1344/RIDAS2018.6.4 


\section{Otras acciones}

La Gerencia de Servicios de Educación también colabora con otros agentes e instituciones para dar a conocer las oportunidades del ApS en la política educativa local. Destacamos la colaboración con otras áreas de la Diputación para generar complicidades y sumar recursos (Medio Ambiente, Parques Naturales, Deportes, Juventud, Bibliotecas...). También la participación en jornadas, como es el caso de las 5as y las 6as Jornadas de intercambio de experiencias de aprendizaje-servicio (2014 y 2017 respectivamente). En las primeras, la Diputación de Barcelona participaba en el Ágora de experiencias conduciendo el grupo de trabajo $L a$ implicación del territorio en el ApS, que hacía hincapié en la idea de que el territorio es el espacio natural de arraigo de los proyectos de aprendizaje-servicio, con el objetivo final de mejorar el éxito educativo. En las segundas, se participó en una mesa redonda con otras instituciones presentando La red y el territorio, donde la reflexión giraba en torno a la importancia de la implicación de la administración en el impulso de propuestas educativas a nivel municipal como los proyectos de aprendizaje servicio y los éxitos logrados en la estrategia propia de la Diputación en este impulso.

\section{Esquema de formulación de una estrategia local de aprendizaje- servicio}

El acompañamiento y apoyo a los ayuntamientos sigue un proceso sistematizado que se adecuará, en la medida de lo posible, a las necesidades específicas de cada municipio. Este proceso incluye: elaboración de un plan de trabajo; elaboración de documentos; trabajo con el referente técnico y/o político; trabajo con entidades; creación de un grupo motor o encaje en otros espacios ya existentes; $y$, momento de cierre del proceso realizado.

Elaboración de un plan de trabajo. Se elabora un plan de trabajo que recoge las actuaciones que, de forma genérica, se llevarán a cabo a lo largo del proceso de apoyo. Estas actuaciones son:

- Mapeo

- Acompañamiento técnico al/los referente/s del proyecto

- Trabajo con técnicos y entidades

- Comunicación del proceso en los centros educativos

- Creación de una comisión, grupo motor, red...

- Encuentro o jornada de valoración

- Cierre: evaluación del proceso realizado e identificación y descripción de retos de futuro

Elaboración de documentos

- Sobre el mapeo: cuadro de recopilación de experiencias y documento de análisis de los resultados del mapeo. Estos documentos permiten un análisis cuidadoso de las actuaciones, agentes y recursos implicados en proyectos con metodología aprendizaje-servicio

- Elaboración de un documento marco técnico-político para la articulación del servicio comunitario y otras actuaciones con metodología ApS en 
la política educativa local. La razón de ser de este documento es la naturaleza transversal de la metodología ApS; muchos de los ámbitos de trabajo de los ayuntamientos se pueden sentir interpelados. El objetivo de este documento es implicar a las diferentes áreas o departamentos del ayuntamiento en esta estrategia.La implicación se hace desde el ámbito político al técnico, desde la concejalía de educación se comparte con el resto de concejalías. Estas lo comunican a los/las resposnables de área o departamento para que puedan identificar a las personas que se implicarán en esta estrategia a nivel técnico. Es importante para el personal técnico que esta implicación se entienda y se visualice como un encargo que pasa a formar parte de su trabajo habitual.

- Otros documentos según necesidad

Trabajo con técnicos $y / o$ representantes políticos municipales

El objetivo de este punto es ofrecer al ayuntamiento las herramientas necesarias para la apropiación de la estrategia. Las acciones (formación, sensibilización...) que se realicen dependerán del grado de conocimiento de los técnicos y/o políticos sobre servicio comunitario y ApS en general.

\section{Trabajo con entidades}

El objetivo de este punto es poner en conocimiento de las entidades educativas del municipio o con actuaciones potencialmente educativas, la estrategia del ayuntamiento para con el servicio comunitario y la metodología ApS, así como visualizar las oportunidades de colaboración en esta estrategia. Las acciones que se realicen dependerán del grado de conocimiento y participación de las entidades en el proyecto Servicio Comunitario y otros proyectos con metodología ApS.

\section{Creación de un grupo motor u otras variantes}

El objetivo de esta actuación es asegurar que existe un punto de referencia para la estrategia de articulación del servicio comunitario y el ApS en la política educativa local. Este punto se creará de nuevo si no existe ningún espacio que lo pueda asumir, o bien se encajará en algún órgano o espacio de trabajo ya existente.

\section{Jornada de reconocimiento/cierre/trabajo}

Es recomendable generar un momento y espacio para cerrar el proceso de trabajo pero también para dialogar sobre cómo se está desarrollando la estrategia Servicio Comunitario-ApS. El énfasis sobre el reconocimiento de proyectos, o sobre el trabajo a fondo de temáticas propias de los proyectos con metodología ApS, u otras opciones que se quieran plantear, dependerá de las necesidades que se identifiquen en cada uno de los municipios y en cada uno de los momentos de desarrollo de la estrategia.

\section{Valoración del proceso realizado}

Valoración de la estrategia de trabajo y

Maroto, S. (2018). La educación se mueve en el territorio. Estrategias locales de aprendizaje-servicio. RIDAS, Revista Iberoamericana de Aprendizaje Servicio, 6, 19-32. DOI10.1344/RIDAS2018.6.4 
apoyo a los entes locales

Esta estrategia ha permitido avanzar de procesos de acompañamiento aislados respecto a la política educativa local para convertirse en un instrumento de desarrollo de la política educativa a nivel municipal. Se ha pasado de poner el foco en las oportunidades metodológicas del ApS a ponerlo en las oportunidades estratégicas de las actuaciones desarrolladas con esta metodología. Se ha incorporado el proyecto Servicio Comunitario como un motor de cambio en esta nueva mirada. La obligatoriedad de ser cursado por todos los alumnos de $3^{\circ}$ o $4^{\circ}$ de ESO a partir del curso desde 2019 hasta 2020 ha generado una nueva oportunidad para poner en valor las posibilidades de apoyo a los procesos de aprendizaje desde la movilización de los recursos del entorno.

Todos estos elementos se han puesto en práctica en dos experiencias piloto:Sant Cugat del Vallès y Puig-reig. Ambas experiencias han finalizado con éxito, logrando ampliamente los objetivos planteados.

En el caso de Puig-reig ha conseguido vincular toda la comunidad educativa y parte de la comunidad vecinal a un solo proyecto con metodología ApS. Desde la concejalía se ha valorado la iniciativa como una experiencia de éxito en la movilización de agentes, en la creación de red y oportunidades de trabajo corresponsable. Actualmente siguen teniendo apoyo de Diputación para la consolidación de la estrategia.

En el caso de Sant Cugat del Vallès los objetivos se plantearon, sobretodo, para la apropiación de la estrategia por parte del ayuntamiento, entendiéndola como un proyecto transversal que requiere la participación de diferentes técnicos y áreas. Objetivo que se ha logrado con bastante éxito y por el que todavía se está trabajando con el apoyo de Diputación.

Otro gran objetivo era ordenar las actuaciones con metodología ApS y conseguir hacer red con el Departamento, a través de los centros de recursos pedagógicos, en el caso del proyecto Servicio Comunitario. Este objetivo también se ha alcanzado en Sant Cugat. Uno de los elementos que ponen de manifiesto este logro es la demanda que el centro de recursos pedagógicos ha hecho en el ayuntamiento para colaborar activamente en las últimas formaciones docentes sobre el proyecto Servicio Comunitario. En el caso de Puig-reig, la dimensión comarcal y no municipal del centro de recursos pedagógicos ha dificultado un diálogo fluido entre ambas instituciones. El objetivo, sin embargo, se mantiene.

\section{Valoración de la suma de acciones}

La suma de actuaciones de apoyo en el ámbito del aprendizaje-servicio, que incluye tanto acciones formativas, participación en jornadas, aportación de conocimientos y reflexiones, como acompañamiento e intervención, han contribuido a crear un conocimiento extendido y compartido en varias poblaciones de la demarcación de Barcelona.

La conclusión principal a la que se llega es que el elemento clave para el arraigo

Maroto, S. (2018). La educación se mueve en el territorio. Estrategias locales de aprendizaje-servicio. RIDAS, Revista Iberoamericana de Aprendizaje Servicio, 6, 19-32. DOI10.1344/RIDAS2018.6.4 
del ApS es que forme parte de la política educativa local. Esto implica dos vertientes fundamentales de acción:

a) Por un lado la vertiente política y técnica. El ApS no puede arraigarse en una sola persona (técnico educación) o en la voluntad y convicción de una o más personas, ya sea a nivel técnico o político. Debe haber una apropiación consciente y expresa del ayuntamiento, una decisión política que reconozca y dimensione el espacio técnico de trabajo en el seno del ayuntamiento. Esto significa identificación de áreas o departamentos, técnicos y estructura de funcionamiento:

- En relación a las áreas, identificar cuáles deben implicarse de forma sistemática y cuáles de forma puntual

- En relación al personal técnico, la participación en la estrategia ApS debe formar parte del trabajo de cada uno de los técnicos $y$, por tanto, debe estar reconocida por los mandos correspondientes

- En cuanto a la estructura de funcionamiento, hay que definir cómo se quiere funcionar (comisión, grupo motor...) e identificar si hay que crear de nuevo o si ya existen espacios de trabajo que pueden encajar esta estrategia (consejo escolar municipal, comisiones transversales de técnicos, planes estratégicos ...)

La dificultad principal que se ha planteado en esta línea de acción es la falta de planificación estratégica en cuanto a política educativa local. Se traduce en la realización de acciones aisladas: formaciones aisladas, intervenciones aisladas, no creación de red ni de espacios para compartir. Esta situación se revierte si desde Diputación se reorienta la demanda hacia la construcción de una estrategia.

También hay que mencionar como dificultad la excesiva dependencia de la motivación y voluntad de los técnicos y otros agentes de la comunidad educativa. Puede ser una chispa pero en ningún caso es suficiente ni debe ser el elemento de sustentación de la estrategia.

b) Por otro lado la vertiente "estrategia de territorio". Si bien la articulación a nivel de ayuntamiento es condición necesaria para el arraigo del ApS, también lo es la articulación en el marco del municipio. Es necesario un compromiso y estructura a nivel de ente local pero también a nivel de territorio: con qué agentes podemos contar, en qué espacios y cómo trabajaremos.

\section{Retos de futuro: ¿qué podemos hacer para seguir mejorando}

Mantener y fortalecer las acciones que nos han funcionado o pueden funcionar bien, como las formaciones, seminarios y comunicación; la realización del apoyo técnico a los entes locales a partir de un proceso sistematizado; crear un grupo de trabajo interáreas para aprovechar todos los recursos de Diputación.

Establecer una relación de trabajo con el Departamento de Educación y otras instituciones, si se da el caso. La poca relación con el Departamento de 
Educación en cuanto al proyecto Servicio Comunitario hace perder la oportunidad de poner en valor la suma de las dos acciones: Proyecto Servicio Comunitario y Estrategia Servicio Comunitario y ApS de la Diputación de Barcelona.

\section{Situar la estrategia en la política} educativa local, no sólo para aquellos ayuntamientos que solicitan apoyo sino para todos aquellos que ponen en valor la participación en los procesos de gobernanza educativa. Es de especial interés vincular el ApS a proyectos y programas como los Planes Educativos de Entorno, los Proyectos Educativos de Ciudad o la Educación 360. Educación a Tiempo Completo.

Sistematizar un proceso de seguimiento y evaluación de las acciones realizadas, para disponer de datos que permitan ajustar la estrategia de intervención de la Diputación de Barcelona.

\section{Para finalizar}

El aprendizaje-servicio es una metodología educativa que, en el marco de posibles propuestas pedagógicas, aporta una singularidad que la hace especialmente atractiva para las administraciones locales: la dimensión del servicio.

Necesita del territorio para poder ser. Siempre puede haber experiencias intra-centro que pasarán por alto este requisito. Pero aun así también los principios pedagógicos de trabajar sobre necesidades reales y para la transformación social nos llevan directamente al entorno. Por regla general el barrio, distrito o municipio se convierte en el aliado imprescindible para que esta práctica educativa se lleve a cabo. El éxito dependerá de la implicación de todos los agentes en este proceso, de la capacidad de generar sinergias y crear red -en el formato que se quiera- que impulse, promueva y apoye estos proyectos.

Por suerte para las políticas educativas locales, el ApS interpela entidades y administraciones a ser corresponsables y a trabajar desde la transversalidad. La riqueza y amplitud que nos propone el aprendizaje-servicio no se puede quedar en el voluntarismo de algunas personas. Es una oportunidad práctica y real de conectar espacios, dimensiones, agentes y participantes. Una oportunidad para ayudar a abrir las paredes de los centros educativos en el entorno y a la inversa, y para poner en valor el potencial educativo de gran parte de las entidades sociales, culturales, ambientales... de nuestros municipios. Su carácter de instrumento de concreción de las políticas educativas locales dota esta metodología de una dimensión operativa complementaria a la de programas más conceptuales y de mayor alcance.

La decisión de la Diputación de continuar trabajando con esta metodología se mantiene, porque la trayectoria vivida lo avala y porque la realidad educativa, con el impulso del servicio comunitario, lo requiere. Se mantiene de forma dinámica, abierta a los cambios y a la vinculación con otros proyectos de participación, gobernanza y mejora del éxito educativo, para acompañar a los entes locales de la 
forma más adecuada en cada momento

\section{Referencias bibliográficas}

Diputación de Barcelona. Área de Educación (2008). Guía metodológica del Aprendizaje-Servicio. Colección Documentos de Trabajo. Serie Educación. Barcelona: Diputación de Barcelona.

Tarabini, A., Bonal, X. y Plandiura, R. (2017). Políticas educativas en Cataluña: competencias, financiación y educación. En Gomà, R. y Subirats, ] (coords). Cambio de época y de políticas públicas en Cataluña.Barcelona: Crítico.

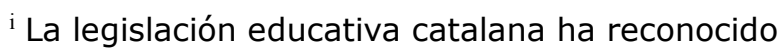
la participación y gestión del mundo local en la prestación de centros y servicios de escolarización en primera infancia (0-3 años) pero se mantiene residual o intermitente en el resto de ámbitos. Para una aproximación al marco competencial local y su ejercicio véase Diputación de Barcelona (2017). Observatorio de la educación local 2016. Barcelona:

Diputación de Barcelona.

ii Las competencias locales en educación son las que los ayuntamientos, como gobierno local, tienen respecto al ámbito de educación. La Diputación de Barcelona, en su función de apoyo a los ayuntamientos, trabaja en el marco de estas competencias.

iii Ley $12 / 2009$, de 10 de julio, de educación (LEC)

iv Marí-Klose, M.; Gómez-Granell, C.; Claveria, S.; Muñoz, A.; Sánchez, L.; Bosch, C. (2010). Acompañamiento a la escolaridad: Pautas para un modelo local de referencia. Área de

Educación. Gerencia de Servicios de Educación. Barcelona: Diputación de Barcelona.

v La Ciudad de Barcelona dispone de un Consorcio de Educación y un marco específico de actuación en materia educativa -Carta Municipal de Barcelona 22/1998-, por lo que la Diputación de Barcelona no trabaja directamente con la ciudad sino con el resto de los 310 municipios de la demarcación de
Barcelona.

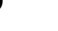

